\title{
Increased expression of CD44 in hypertrophied ligamentum flavum and relevance of splice variants CD44v5 and CD44v6
}

\author{
Stefan Lakemeier • Raphael Schmid • Lisa Foltz • \\ Jochen Rohlfs • Susanne Fuchs-Winkelmann • \\ Turgay Efe • Christian Foelsch • Jürgen R. J. Paletta
}

Received: 14 July 2011 / Accepted: 7 October 2011 /Published online: 4 November 2011

(C) Springer-Verlag 2011

\begin{abstract}
Background The most common spinal disorder in the elderly is lumbar spinal stenosis (LSS), which results in part from ligamentum flavum (LF) hypertrophy. Although prior histologic and immunochemical studies have been performed in this area, the pathophysiology of loss of elasticity and hypertrophy is not completely understood. The purpose of this immunohistological study is to elucidate the role of CD44 and its splice variants CD44v5 and CD44v6 in the hypertrophied LF obtained from patients with lumbar spinal stenosis (LSS).

Materials and methods LF samples of 38 patients with LSS were harvested during spinal decompression. Twelve LF samples obtained from patients with disc herniation and no visible degeneration on preoperative MRI were obtained as controls. Samples were dehydrated and embedded in paraffin. For immunohistochemical determination, slices were stained with antibodies against CD44, Cd44v4, and CD44v6 stained with DAB. LF hypertrophy and cross-sectional area (CSA) were measured with T1-weighted MRI.

Results CD44 and CD44v5 expression were significantly increased in the hypertrophy group $(p<0.05)$. CD44v6
\end{abstract}

Christian Foelsch and Jürgen RJ Paletta contributed equally to this study.

S. Lakemeier $(\bowtie) \cdot$ R. Schmid $\cdot$ L. Foltz $\cdot$

S. Fuchs-Winkelmann • T. Efe • C. Foelsch • J. R. J. Paletta

Department of Orthopedics and Rheumatology,

University Hospital Marburg,

Baldingerstrasse,

35043 Marburg, Germany

e-mail: lakemeie@med.uni-marburg.de

J. Rohlfs

Department of Neurosurgery, University Hospital Marburg,

Marburg, Germany expression was not significantly increased. The number of elastic fibers was significantly higher in the hypertrophy group. In the hypertrophy group, LF thickness was significantly increased while CSA was significantly decreased. There was a statistical correlation between LF thickness, CSA, CD44, and CD44v5 expression in the hypertrophy group $(p<0.05)$.

Conclusions LF hypertrophy is accompanied by increased CD44 and CD44v5 expression. CD44v6 expression is not enhanced in LF hypertrophy.

Keywords Lumbar spinal canal stenosis · CD44 - CD44v5 · CD44v6 - Ligamentum flavum hypertrophy Elastic fiber number

\section{Introduction}

Hypertrophy of the ligamentum flavum (LF) is an important contributing factor in lumbar spinal stenosis (LSS), lower back pain, as well as sciatica [31]. In the normal LF, $60-70 \%$ of the extracellular matrix consists of elastic fibers, whereas hypertrophied LF demonstrates an increase in collagen fibers, calcification, ossification, and chondrometaplasia. As these age-related degenerative changes progress, LF tissues become disorganized, contributing to LSS development [17, 29, 32, 36, 43, 45].

LF thickening can be measured on axial T1-weighted magnetic resonance imaging (MRI). LF thickening appears to be age-dependent and gender-independent. Abbas et al. and Sakamaki et al. described a significantly thickened LF in LSS versus healthy patients $[1,35]$. The cross-sectional area (CSA) of the cauda equina measured in MRI axial cuts can be directly related to clinical symptoms [28]. 
Previous studies have primarily investigated LF histological changes. Fibrosis seems to be one of the contributing factors of LF hypertrophy as in hypertrophied LF samples a relationship between scar tissue formation and LF thickness was shown [34].

CD44 is a polymorphic type I transmembrane glycoprotein [39]. During tendon fibrotic healing, it functions as a key mediator. It is involved in cell-cell and cell-matrix interactions, cellular migration, extracellular matrix assembly, as well as cytokine binding and activation [20,27]. Its most important function seems to mediate the uptake and clearance of hyaluronic acid (HA) [5]. It was shown that high levels of HA are beneficial for tendon healing [42]. During scarless fetal healing, high levels of HA and downregulated levels of CD44 have been observed [11]. As HA is mainly degraded by CD44, a connection between scar tissue formation in tendon healing and levels of CD44 expression seems to be likely. CD44 is the standard isoform of the molecule $[19,24]$. Although CD44 is mainly expressed, several alternatively spliced variants of CD44 have been described in the literature (splice variants v1-10) [38]. The expression of CD44v6 is correlated with inflammatory diseases such as rheumatoid arthritis, whereas CD44v5 is thought to be involved in tumor progression and osteoarthritis $[14,16]$.

The expression of CD44 and its splice variants CD44v5 and CD44v6 during LF hypertrophy have not been evaluated until now.

The aims of our study were (1) to show an increased expression of CD44 and its splice variants CD44v5 and CD44v6 hypertrophied LF compared with healthy controls, and (2) to find a potential correlation between CD44 expression, LF thickness, and CSA.

\section{Materials and methods}

\section{Patients}

Fifty patients (29 male, 21 female) were prospectively included in our study. Study approval was obtained from our institutional ethics committee. Thirty-eight patients with LSS necessitating decompressive surgery comprised the study group; LF specimens were harvested from the L4/L5 spinal level. The control group consisted of 12 patients with disc herniation at the L4/L5 level. None of the controls had signs of degeneration on preoperative MRI.

\section{Radiographic assessment}

All patients underwent preoperative MRI of the lumbar spine. Cauda eqina CSAs were determined by both an experienced radiologist and an experienced spine surgeon.
The cauda eqina CSA at the L4/L5 level was determined on axial T1-weighted images using a picture analyzing system (PACS, Agfa-Gevaert group, Mortsel, Belgium). LF thickness was measured retrospectively on axial MRI T1-weighted images obtained at the L4/L5 facet joint of each patient $[30,33]$.

\section{Specimen preparation}

LF samples were immediately fixed in $4 \%$ formaldehyde for $24 \mathrm{~h}$, dehydrated in graded alcohol solution and cedarwood oil, and then embedded in paraffin. Samples were cut into $5-\mu \mathrm{m}$ sections with a Leica microtome RM2055 (Leica, Bensheim, Germany) $40^{\circ}$ stainless-steel knife. Histologic standard staining was performed with a Masson-Goldner staining kit (Merck, Darmstadt, Germany) according to the manufacturer's instructions.

\section{Immunochemistry}

For immunohistologic staining of CD44, CD44v5, and CD44v6, slices were rehydrated and incubated in citrate buffer (pH 6) at $90^{\circ} \mathrm{C}$ over $10 \mathrm{~min}$. After blocking with normal horse serum, the slices were incubated overnight $\left(4^{\circ} \mathrm{C}\right.$, humidified chamber) with the following monoclonal antibodies: CD44 clone SFF-2 mouse, dilution 1:100 (Bender, Vienna, Austria); CD44v5 clone VFF-8 mouse dilution 1:50 (Bender, Vienna, Austria), CD44v6 clone VFF-18, mouse, dilution 1:100 (Bender, Vienna, Austria). Immunostaining was performed either using a labeled streptavidin-biotin method (Dako, Hamburg, Germany, REAL Detection System Peroxidase/DAB+). The staining reaction based on 3,3'-diaminobenzidine (DAB) as the substrate (Vector Laboratories, Burlingame, CA). The stained slices were rinsed with distilled water and stained for $15 \mathrm{~s}$ with hematoxylin and eosin as a counterstain. Finally, the sections were rinsed with water and treated with graduated density alcohol and xylol [23]. Examples of typical stained LF sections are given in Fig. 1a-d.

\section{Histology}

Histomorphometric analysis was performed at a primary objective lens magnification of $5 \times$ using a Leica DM5000 and Quips analysis software (Leica, Bensheim, Germany). In order to characterize cells and to perform a cell count, a $40 \times$ objective lens magnification was used. For differential stained slices (e.g., Masson Goldner stained slices), a 10× objective lens magnification was used. Elastic fiber number was determined by counting and measuring elastic fibers in ten different areas of every specimen. The values indicated are the mean values for elastic fiber number in the counted 
Fig. 1 a-d Ligamentum flavum sections after DAB staining and incubation with different antibodies ( $40 \times$ objective lens magnification. In hypertrophied LF sections, high expression of CD44 (a), CD44v5 (c) and little vessel-associated CD44v6 expression (d) is visible. In the control group (b), CD44 expression is low
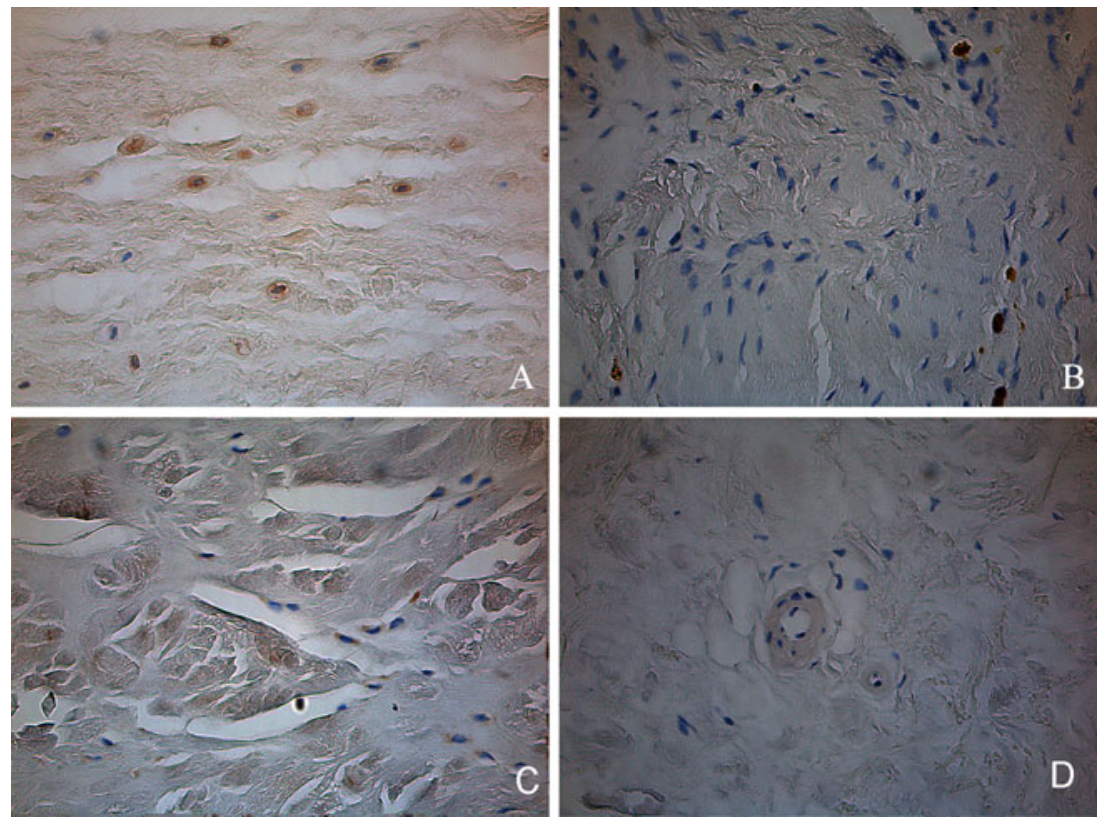

ten different areas. Cell counting of immunohistochemically stained slices was performed by a $40 \times$ objective lens magnification. The percentage stands for the quotient of CD44, CD44v5, and CD44v6-positive cells in relation to the total number of cells per slice (Fig. 1a-d).

\section{Statistics}

Student's $t$ test was used to evaluate the differences between the experimental and control sites and between the different groups. Data are given as means \pm standard error of measurements [44]. Correlations between CD44, CD44v5, CD44v6, elastic fibers, LF thickness; and CSA were calculated according to Pearson. The level of significance was set at $p<0.05$.

\section{Results}

Patient demographics

The mean age in the hypertrophy group was 60.4 (range 50-85) years, and 48.8 (range 20-63) years in the control group (Table 1).

\section{Radiographic assessment}

CSA and LF thickness were significantly increased in the hypertrophy group $(p<0.05)$. A significant correlation between CD44 and CD44v5 expression, CSA, and LF thickness could be found $(p<0.05)$. There was no significant correlation between patient age and LF thickness and CSA (Table 1, Fig. 2a).
CD44, CD44v5, and CD44v6 expression and elastic fibers

CD44 and CD44v5 expression was significantly increased in the hypertrophy versus control group $(p<0.05)$. CD44v6 expression was not significantly increased and strictly associated to the small number of vessels that were found in the dorsal parts of both hypertrophied and healthy LF. There was no correlation between age, gender, and CD44 as well as CD44v5 expression in the hypertrophy nor in the control group $(p<0.05)$ whereas in overall patients, a correlation between age and the expression of these molecules could be described ( $p>0.05$, Fig. 2a). The number of elastic fibers was significantly decreased in the hypertrophy group ( $p<0.05$, Table 1, Fig. $2 b$ ).

\section{Discussion}

This is the first study to evaluate the expression of CD44 and its splice variants CD44v5 and CD44v6 in hypertrophied LF samples. We could show a significantly increased expression of CD44 and CD44v5 whereas the expression of CD44v6 was not significantly elevated. Our results lead to the conclusion that CD44 has an important function during LF hypertrophy. So far, the expression of CD44 has been extensively studied on tendons and in tendon healing. Ansorge et al. could show that CD44 knock-out mice tendon healing was improved compared to wild-type mice. The authors postulate that altering the activity of the CD44 receptor offers multiple advantageous effects and leads to superior material properties [4]. Previous studies showed an over-expression of CD44 in fibrotic healing and a decreased expression of the factor in scarless healing [11]. 

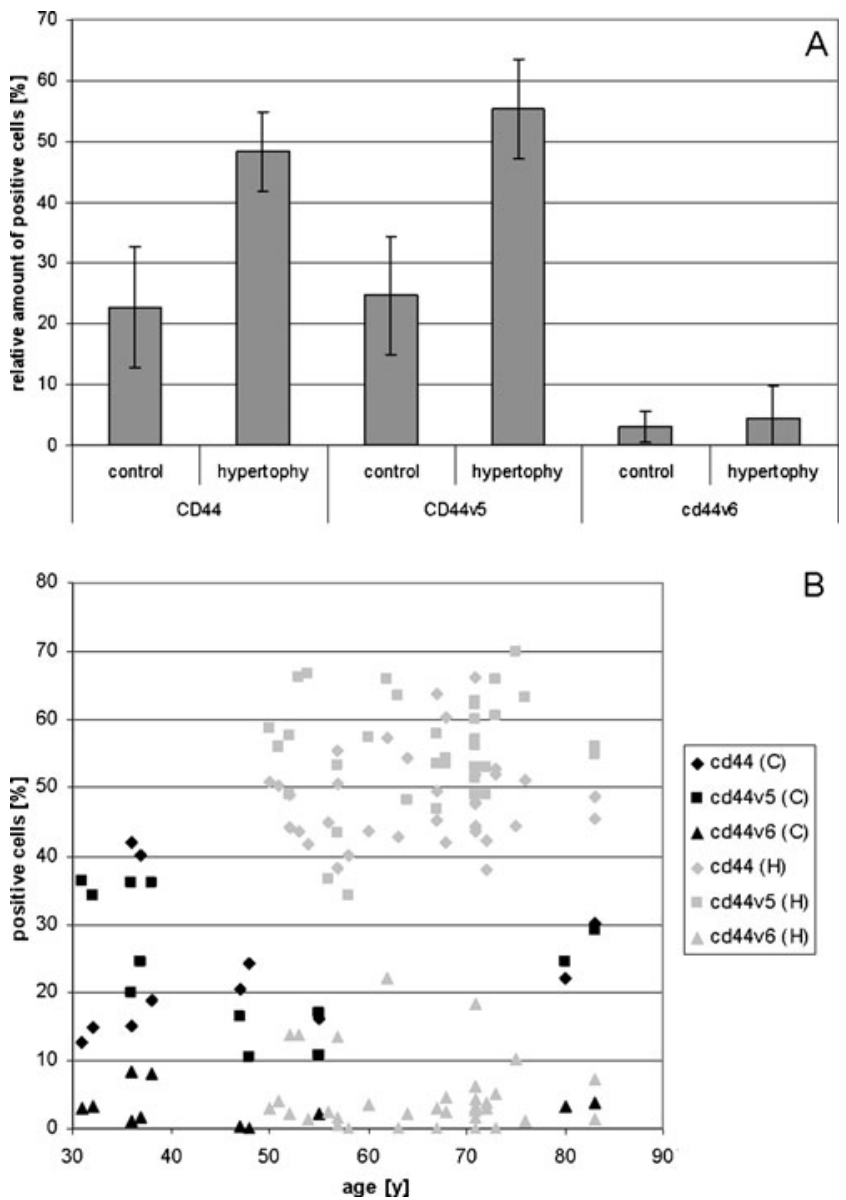

Fig. 2 a-b Mean values for CD44, CD44v5, and CD44v6 expression in hypertrophy and control group (a) and correlation of patient age as well as expression of CD44, CD44v5, and CD44v6 in the hypertrophy and control group (b)

Furthermore, it was shown that HA plays a pivotal role in tendon healing [22]. This glycosaminoglycan is abundant in tendons and studies have proven, that in healing fetal tendons, HA levels surpass those in healing adult ones [2]. Park et al. showed high levels of "Transforming Growth Factor Beta 1" (TGF- $\beta 1$ ) in hypertrophied LF samples [30]. TGF- $\beta 1$ is a factor that stimulates fibroblasts and chondrocytes. Thus, it contributes to pathologic tissue fibrosis $[9,12]$. Furthermore, enhanced collagen 1 expression plays a role in LF hypertrophy [25]. In vitro, the expression of TGF- $\beta 1$ and collagen 1 could be enhanced by the addition of HA, but these effects were no longer present when the cells were pretreated with CD44-silencing RNA [10].

Another contributing factor to LF hypertrophy is the expression of matrix metalloproteinases (MMP). MMPs play a key role in the degradation of extracellular matrix and in intercellular communication, cell migration, tumor progression, and angiogenesis [3]. Elevated MMP 2, 3, and 13 expression in LF samples obtained from patients with LSS have been reported by Park et al. and elevated MMP 3 
expression by $\mathrm{Oh}$ and $\mathrm{Ha}[30,34]$. In vitro, MMP expression is inhibited by the addition of HA via CD44 [18]. However, the role of HA during LF hypertrophy needs to be elucidated. There is some evidence that modulation of CD44 activity via HA may offer a novel therapeutic option for early LSS. Our findings would be helpful in this regard; i. e., it could be demonstrated that addition of sodium hyaluronate to the remaining LF after discectomy can preserve adhesions between dura and LF [21].

Mechanical loading seems to be one etiological factor for CD44 expression. In cultivated rat tail derived tendon fibroblasts, Crockett et al. found a greater than $60 \%$ increase of CD44 and a twofold increase of HA expression after cyclic mechanical loading of the cells [7].

We showed that CD44 expression occurs mainly in the dorsal LF portion, which is subjected to more mechanical loading than the dural portion [33].

Another etiological factor could be Interleukin 1 (IL1). After treatment with IL1 human chondrocytes showed a proteoglycan loss and enhanced expression of CD44 [26]. Sairyo et al. demonstrated a correlation between IL1 expression and thickness of the hypertrophied LF [34]. Thus, LF hypertrophy could be a reaction to either mechanical or chemical stimuli. Furthermore, some evidence is available that CD44 expression is related to age. Zhou et al. found that aged rat tendon-derived stem cells expressed higher levels of CD44 than young ones [46]. Thus, while age may be a factor in our findings, we do not consider it to be the main explanation, since we were unable to demonstrate a significant correlation between age and CD44 expression and LF thickness in the hypertrophy group. Therefore, the reason for CD44 expression remains unclear at this time.

The splice variants CD44v6-v7, CD44v5-v9, and CD44v2-v10 in the synovial fluid of osteoarthritic patients were first identified by Hale et al. [15]. One explanation for the increased CD44v5 expression can be found in its altered binding capacity compared to CD44. In tumor cells, a correlation between increased ectopic expression of CD44v5-7 on the cell surface and increased soluble HAbinding capacity was observed [37]. The authors postulated that the regulation of CD44 clustering is mediated by factors such as the presence of variant exons and glycosylation and allows the cells to regulate their HAbinding capacities. Therefore, CD44 splice variant expression might be seen as a response mechanism. In previous studies, a correlation between CD44v5 expression and histological matrix destruction was seen in osteochondrosis [13]. As we found a significant correlation between elastic fiber number decrease and CD44v5 expression, our results suggest that CD44v5 is also synthesized during LF hypertrophy and participates in the pathogenesis of the disease.
CD44v6 has been demonstrated in synovial fibroblasts of patients suffering from rheumatoid arthritis, osteoarthritis, and autoimmune diseases $[6,8,40]$. Furthermore, it has been shown that lymphocyte activation is accompanied by enhanced CD44v6 expression [41]. In LF samples, CD44v6 expression was strongly associated with vessels found in the dorsal LF portion and might be explained by the remaining lymphocytes in the vascular walls. However, expression of this factor was low in both the hypertrophy and the control group indicating that CD44v6 expression does not play an important role in LF hypertrophy.

The measurement of LF thickness on T1-weighted MRIs and CSA on MRI or CT scans are approved procedures in LSS diagnosis. In the LSS group, LF was significantly thickened and CSA was significantly less compared to controls, also confirming previous reports [1, 28, 35].

In summary, we could show that CD44 expression is correlated with LF thickness and CSA. Together with the results of the literature, our findings indicate that CD44 plays a pivotal role in the cellular process leading to LF hypertrophy, although the molecular mechanism is not completely clarified.

Our study has several limitations. First, our control group was small (12 patients) compared to the hypertrophy group. As it was our goal to compare hypertrophied and normal LF, we only used LF samples from patients with an indication for discectomy and no visible signs of degenerative lumbar spine disease - a rare indication.

LSS is associated with different levels of osteochondrosis of the facet joints, which may influence CD44 expression in the LF. Therefore, LF samples were only obtained from the midportion of the LF, between connected vertebral laminae. Furthermore, we only obtained L4/L5 samples to ensure that the study groups would be comparable. Third, there was a difference in mean age between the study and control groups. This may partly explain our results. As we did not find a statistically significant correlation between mean age and LF hypertrophy, especially in the hypertrophy group, we do not believe that normal aging is the main explanation for our findings. However, age is a known etiological factor for LSS. Nevertheless, even if it were the most important contributing factor for CD44 expression, the potential positive benefit of our results in LSS treatment remains unchanged.

\section{Conclusions}

We demonstrated that LF hypertrophy is associated with increased expression of CD44 and the splice variant CD44v5. CD44v6 does not contribute to LF hypertrophy. Our study provides a molecular mechanism for biomechan- 
ical changes observed in LSS that are not explained by morphologic characteristics alone.

\section{Conflicts of interest None.}

\section{References}

1. Abbas J, Hamoud K, Masharawi YM, May H, Hay O, Medlej B, Peled N, Hershkovitz I (2010) Ligamentum flavum thickness in normal and stenotic lumbar spines. Spine (Phila Pa 1976) 35 (12): $1225-1230$

2. Adzick NS, Longaker MT (1992) Scarless fetal healing. Therapeutic implications. Ann Surg 215(1):3-7

3. Akhavani MA, Madden L, Buysschaert I, Sivakumar B, Kang N, Paleolog EM (2009) Hypoxia upregulates angiogenesis and synovial cell migration in rheumatoid arthritis. Arthritis Res Ther 11(3):R64

4. Ansorge HL, Beredjiklian PK, Soslowsky LJ (2009) CD44 deficiency improves healing tendon mechanics and increases matrix and cytokine expression in a mouse patellar tendon injury model. J Orthop Res 27(10):1386-1391

5. Aruffo A, Stamenkovic I, Melnick M, Underhill CB, Seed B (1990) CD44 is the principal cell surface receptor for hyaluronate. Cell 61(7):1303-1313

6. Baaten BJ, Li CR, Bradley LM (2010) Multifaceted regulation of $\mathrm{T}$ cells by CD44. Commun Integr Biol 3(6):508-512

7. Crockett RJ, Centrella M, McCarthy TL, Grant Thomson J (2010) Effects of cyclic strain on rat tail tenocytes. Mol Biol Rep 37 (6):2629-2634

8. Croft DR, Dall P, Davies D, Jackson DG, McIntyre P, Kramer IM (1997) Complex CD44 splicing combinations in synovial fibroblasts from arthritic joints. Eur J Immunol 27(7):1680 1684

9. Cunliffe IA, Rees RC, Rennie IG (1996) The effect of TGF-beta 1 and TGF-beta 2 on the proliferation of human Tenon's capsule fibroblasts in tissue culture. Acta Ophthalmol Scand 74(1):31-35

10. David-Raoudi M, Tranchepain F, Deschrevel B, Vincent JC, Bogdanowicz P, Boumediene K, Pujol JP (2008) Differential effects of hyaluronan and its fragments on fibroblasts: relation to wound healing. Wound Repair Regen 16(2):274-287

11. Favata M, Beredjiklian PK, Zgonis MH, Beason DP, Crombleholme TM, Jawad AF, Soslowsky LJ (2006) Regenerative properties of fetal sheep tendon are not adversely affected by transplantation into an adult environment. J Orthop Res 24 (11):2124-2132

12. Franzen L, Dahlquist C (1994) The effect of transforming growth factor-beta on fibroblast cell proliferation in intact connective tissue in vitro. In Vitro Cell Dev Biol Anim 30A(7):460-463

13. Fuchs S, Dankbar B, Wildenau G, Goetz W, Lohmann $\mathrm{CH}$, Tibesku CO (2004) Expression of the CD44 variant isoform 5 in the human osteoarthritic knee joint: correlation with radiological, histomorphological, and biochemical parameters. J Orthop Res 22 (4):774-780

14. Fuchs S, Rolauffs B, Arndt S, Tibesku CO, Prehm P (2003) $\mathrm{CD} 44 \mathrm{H}$ and the isoforms CD44v5 and CD44v6 in the synovial fluid of the osteoarthritic human knee joint. Osteoarthr Cartil 11 (12):839-844

15. Hale LP, Haynes BF, McCachren SS (1995) Expression of CD44 variants in human inflammatory synovitis. J Clin Immunol 15 (6):300-311
16. Heyse TJ, Malcherczyk D, Moll R, Timmesfeld N, Wapelhorst J, Fuchs-Winkelmann S, Paletta JR, Schofer MD (2010) CD44: survival and metastasis in chondrosarcoma. Osteoarthr Cartil 18 (6):849-856

17. Ivancic PC, Coe MP, Ndu AB, Tominaga Y, Carlson EJ, Rubin W, Dipl-Ing FH, Panjabi MM (2007) Dynamic mechanical properties of intact human cervical spine ligaments. Spine J 7(6):659-665

18. Julovi SM, Ito H, Nishitani K, Jackson CJ, Nakamura T (2010) Hyaluronan inhibits matrix metalloproteinase-13 in human arthritic chondrocytes via CD44 and P38. J Orthop Res 29(2):258-264

19. Kaya G, Rodriguez I, Jorcano JL, Vassalli P, Stamenkovic I (1997) Selective suppression of CD44 in keratinocytes of mice bearing an antisense CD44 transgene driven by a tissue-specific promoter disrupts hyaluronate metabolism in the skin and impairs keratinocyte proliferation. Genes Dev 11(8):996-1007

20. Laurent TC, Fraser JR (1992) Hyaluronan. FASEB J 6(7):23972404

21. Lin HB, Dai JH, Wu XW, Zheng XH, Wang H, Niu SS, Wu JQ, Xu ZX (2007) Prevention of dura adherence in spinal canal after microendoscopic discectomy by different methods: a clinical study of 165 cases. Zhonghua Yi Xue Za Zhi 87(43):3085-3087

22. Lovvorn HN 3rd, Cass DL, Sylvester KG, Yang EY, Crombleholme TM, Adzick NS, Savani RC (1998) Hyaluronan receptor expression increases in fetal excisional skin wounds and correlates with fibroplasia. J Pediatr Surg 33(7):1062-1069, discussion $1069-1070$

23. Matsui Y, Maeda M, Nakagami W, Iwata H (1998) The involvement of matrix metalloproteinases and inflammation in lumbar disc herniation. Spine (Phila Pa 1976) 23(8):863-868, discussion 868-869

24. Miyake K, Medina KL, Hayashi S, Ono S, Hamaoka T, Kincade PW (1990) Monoclonal antibodies to Pgp-1/CD44 block lymphohemopoiesis in long-term bone marrow cultures. J Exp Med 171 (2):477-488

25. Nakatani T, Marui T, Hitora T, Doita M, Nishida K, Kurosaka M (2002) Mechanical stretching force promotes collagen synthesis by cultured cells from human ligamentum flavum via transforming growth factor-beta1. J Orthop Res 20 (6):1380-1386

26. Nishida Y, D'Souza AL, Thonar EJ, Knudson W (2000) Stimulation of hyaluronan metabolism by interleukin-1alpha in human articular cartilage. Arthritis Rheum 43(6):1315-1326

27. Noble PW (2002) Hyaluronan and its catabolic products in tissue injury and repair. Matrix Biol 21(1):25-29

28. Ogikubo O, Forsberg L, Hansson T (2007) The relationship between the cross-sectional area of the cauda equina and the preoperative symptoms in central lumbar spinal stenosis. Spine (Phila Pa 1976) 32(13):1423-1428, discussion 1429

29. Okuda T, Baba I, Fujimoto Y, Tanaka N, Sumida T, Manabe H, Hayashi Y, Ochi M (2004) The pathology of ligamentum flavum in degenerative lumbar disease. Spine (Phila Pa 1976) 29 (15):1689-1697

30. Park JB, Chang H, Lee JK (2001) Quantitative analysis of transforming growth factor-beta 1 in ligamentum flavum of lumbar spinal stenosis and disc herniation. Spine (Phila Pa 1976) 26(21):E492-E495

31. Ploumis A, Transfledt EE, Denis F (2007) Degenerative lumbar scoliosis associated with spinal stenosis. Spine J 7(4):428-436

32. Postacchini F, Gumina S, Cinotti G, Perugia D, De Martino C (1994) Ligamenta flava in lumbar disc herniation and spinal stenosis. Light and electron microscopic morphology. Spine (Phila Pa 1976) 19(8):917-922

33. Sairyo K, Biyani A, Goel V, Leaman D, Booth R Jr, Thomas J, Gehling D, Vishnubhotla L, Long R, Ebraheim N (2005) Pathomechanism of ligamentum flavum hypertrophy: a multidisciplinary investigation based on clinical, biomechanical, histolog- 
ic, and biologic assessments. Spine (Phila Pa 1976) 30(23):26492656

34. Sairyo K, Biyani A, Goel VK, Leaman DW, Booth R Jr, Thomas J, Ebraheim NA, Cowgill IA, Mohan SE (2007) Lumbar ligamentum flavum hypertrophy is due to accumulation of inflammation-related scar tissue. Spine (Phila Pa 1976) 32(11): E340-E347

35. Sakamaki T, Sairyo K, Sakai T, Tamura T, Okada Y, Mikami H (2009) Measurements of ligamentum flavum thickening at lumbar spine using MRI. Arch Orthop Trauma Surg 129(10):1415-1419

36. Schrader PK, Grob D, Rahn BA, Cordey J, Dvorak J (1999) Histology of the ligamentum flavum in patients with degenerative lumbar spinal stenosis. Eur Spine J 8(4):323-328

37. Sleeman J, Rudy W, Hofmann M, Moll J, Herrlich P, Ponta H (1996) Regulated clustering of variant CD44 proteins increases their hyaluronate binding capacity. J Cell Biol 135 (4): 1139-1150

38. Stamenkovic I, Aruffo A, Amiot M, Seed B (1991) The hematopoietic and epithelial forms of CD44 are distinct polypeptides with different adhesion potentials for hyaluronatebearing cells. EMBO J 10(2):343-348

39. Tammi R, Pasonen-Seppanen S, Kolehmainen E, Tammi M (2005) Hyaluronan synthase induction and hyaluronan accumulation in mouse epidermis following skin injury. J Invest Dermatol 124(5):898-905

40. Tibesku CO, Szuwart T, Ocken SA, Skwara A, Fuchs S (2005) Increase in the expression of the transmembrane surface receptor CD44v6 on chondrocytes in animals with osteoarthritis. Arthritis Rheum 52(3):810-817

41. Wittig B, Seiter S, Schmidt DS, Zuber M, Neurath M, Zoller M (1999) CD44 variant isoforms on blood leukocytes in chronic inflammatory bowel disease and other systemic autoimmune diseases. Lab Invest 79(6):747-759
42. Yagishita K, Sekiya I, Sakaguchi Y, Shinomiya K, Muneta T (2005) The effect of hyaluronan on tendon healing in rabbits. Arthroscopy 21(11):1330-1336

43. Yahia H, Drouin G, Maurais G, Garzon S, Rivard CH (1989) Degeneration of the human lumbar spine ligaments. An ultrastructural study. Pathol Res Pract 184(4):369-375

44. Yildirim Y, Kara H, Cabukoglu C, Esemenli T (2006) Suture holding capacity of the Achilles tendon during the healing period: an in vivo experimental study in rabbits. Foot Ankle Int 27 (2):121-124

45. Yoshida M, Shima K, Taniguchi Y, Tamaki T, Tanaka T (1992) Hypertrophied ligamentum flavum in lumbar spinal canal stenosis. Pathogenesis and morphologic and immunohistochemical observation. Spine (Phila Pa 1976) 17(11):1353-1360

46. Zhou Z, Akinbiyi T, Xu L, Ramcharan M, Leong DJ, Ros SJ, Colvin AC, Schaffler MB, Majeska RJ, Flatow EL, Sun HB (2010) Tendon-derived stem/progenitor cell aging: defective selfrenewal and altered fate. Aging Cell 9(5):911-915

\section{Comment}

The authors described over-expression of CD44 in the hypertrophic ligamentum flavum of the lumbar canal stenosis. Their data suggest that the alteration is specifically linked to the hypertrophy of LF, rather than an epi-phenomenon related to the general aging process. This finding would help to advance insight of the pathophysiology of the disease, which is all commonly seen in the elderly population but by no means uniformly affecting them.

Phyo Kim

Tochighi, Japan 